\title{
¿Qué sabemos y qué nos falta estudiar sobre agentes infecciosos transmitidos por garrapatas en Costa Rica?
}

Gaby Dolz ${ }^{1} \bowtie$, Alexander Barrantes González ${ }^{1}$, Marta C. Bonilla ${ }^{1}$, Liliana Campos ${ }^{1}$, Laura Bouza Mora $^{1}$, Mauricio Jiménez Soto ${ }^{1}$, Juan José Romero Zúñiga ${ }^{1}$, Ana E. Jiménez ${ }^{1}$

1 Escuela de Medicina Veterinaria, Universidad Nacional, Heredia, Costa Rica. Email: gaby.dolz.wiedner@una.cr, avbarrantesgonzalez@gmail.com, mabongo27@gmail.com, lilinanac@yahoo.com, laura.bouza.mora@una.cr, drmjimenezsoto@hotmail.com, juan.romero.zuniga@una.cr, ana.jimenez.rocha@una.cr

Las condiciones propias de un país tropical como Costa Rica representan grandes ventajas para la distribución y actividad de las garrapatas, así como facilita que exista una serie de hospedadores para este tipo de ectoparásitos. Ambos factores inciden en la presentación y prevalencia de enfermedades vectoriales zoonóticas. En el presente trabajo se presentan los principales resultados obtenidos en las investigaciones realizadas durante los últimos diez años en la Escuela de Medicina Veterinaria, en las cuales se ha determinado la presencia, se ha estimado la prevalencia y se ha establecido la importancia, en la salud pública, de agentes rickettsiales (Ehrlichia spp., Anaplasma spp. y Rickettsia spp.), piroplasmas (Babesia spp., Theileria spp.), y Hepatozoon canis en animales domésticos (caninos, felinos, equinos y ovinos), animales silvestres (mapaches, pizotes, venados cola blanca) y garrapatas de nuestro país. Además, se presentan los resultados obtenidos en un estudio serológico de donadores de sangre; en especial, la identificación de un nuevo genotipo de Ehrlichia canis hallado en ellos. Finalmente, se discute sobre la diseminación, transmisión y curso de las diferentes enfermedades, como también sobre el diagnóstico, el tratamiento y los estudios que hacen falta para establecer las medidas de control apropiadas a adoptar para el control de estas enfermedades en nuestro país.

凶 Autor para correspondencia Gaby Dolz: gaby.dolz.wiedner@una.cr 